some extent on results from women with both mild and moderate dyskaryosis. In particular, Soutter and Fletcher's overview made no comparison between surveillance and immediate colposcopy ${ }^{2}$; this only confirms that women with some degree of dyskaryosis have a relatively high rate of invasive disease. $^{2}$

The high default rate found by Flannelly et al in Aberdeen is worrying for those continuing a policy of surveillance. This may be due to administrative problems or women's lack of appreciation of the importance of their result. Perhaps women are too frightened to return; perhaps they are too complacent. Either way, our verbal and written presentations of the situation have to some degree influenced this.

What do the general practitioners and the practice nurses who do the counselling think? Local experience with worried women reveals that it is difficult to find appropriate language to express minor degrees of abnormality; this therefore needs further research. Finally, what would the women think of our deliberations? Armed with the facts, which would they prefer? How should we present risk levels, and what is an acceptable level of risk anyway? Perhaps we should ask a sample of women and let this inform the debate.

A rare opportunity exists to show the generalisability of the Aberdeen trial findings. A multicentre, practice based pragmatic trial could include an economic appraisal and consideration of interventions to reduce psychological impact. In the meantime, women's views should be sought, and all those who run the programme at the grass roots level should be fully informed about local decisions by their director of cytology screening.

CLARE WILKINSON

Department of General Practice,

Senior lecturer in general practice

University of Wales College of Medicine,

Llanedeyrn Health Centre,

Llanedeyrn,

Cardiff CF3 7PN

TIM PETERS

Department of Epidemiology and

Public Health Medicine,

Bristol BS8 2PR

1 Flannelly G, Anderson D, Kitchener HC, Mann EMF, Campbell

$\mathrm{M}$. Fisher $\mathrm{P}$, et al. Management of women with mild and $M$, Fisher $P$, et al. Management of women with mild and mode

2 Soutter WP, Fletcher A. Invasive cancer of the cervix in women with mild dyskaryosis followed up cytologically. BMF 1994; 308: 1421-3. (28 May.)

3 Duncan ID. Guidelines for clinical practice and programme management. Oxford: NHSCSP Applied Epidemiology Publications, 1992

\section{No easy answer}

EDITOR,-We are told that well organised screening programmes have worked (British Columbia, Aberdeen, ${ }^{1}$ Iceland), but at the same time change is advocated in most aspects of screening policy (primary screening method, screening interval, threshold for proceeding to treatment). Why? Is poor organisation really the problem with screening or isn't it?

We have struggled in Bristol to provide the best possible cervical screening service: computerised recall since 1977, computerised call up in the 1980 s, an accurate database, $90 \%$ uptake in women under 35 before 1988 and in all eligible women aged 20-64 after 1988, laboratory quality control, training in primary care, improved information for women. Numbers of deaths in Bristol before and after screening show considerable random variation year on year (in a population larger than that of Iceland or Aberdeen) and an age-specific pattern that is consistent with Sasieni's analysis for England and Wales. ${ }^{2}$ This is not the hoped for eradication of deaths and, given the complex cohort time trends that pre-dated screening, nothing that a self respecting sixth form biologist would call firm evidence for screening reducing mortality.

The recently proposed change in management of mild dyskaryosis ${ }^{34}$ has been at issue for some time. We can understand why gynaecologists would rather give immediate diagnosis and treatment for mild dyskaryosis. We deliberately reversed our lowered threshold for referral' because of anxiety caused through waiting for colposcopy. Even a small change in number of referrals makes the difference between queue and no queue, and if we advocate immediate treatment for mild dyskaryosis then similar arguments apply for borderline change, which is far more common. Better to tell women they have minor changes that could be transient, needing a future smear to check and see, than to tell them they need to see a specialist but leave them waiting.

In Flannelly et al's study $32 \cdot 8 \%$ of women with mild and moderate dyskaryosis who had 24 months of surveillance could be told at the end that their cervix was normal compared with $15.4 \%$ of those given immediate treatment. ${ }^{3}$ Is it not worth trying to minimise the number who believe themselves to have "needed treatment for early cancer"? Or is the fear engendered by an abnormal smear result really helped only by seeing a specialist? If the latter is the case then we should be seriously worried, since one in 20 young women who are screened are told that their smear test shows "cellular changes" (dyskaryosis or borderline change).

Greater investment in colposcopy will no doubt be advocated as the solution. Yet how do we ask our purchasing authority to increase the $£ 1.2 \mathrm{~m}$ a year devoted to cervical screening in Bristol when we cannot be sure what the existing effectiveness is, nor what this change will achieve? A E RAFFLE
Consultant in public health medicine

Bristol and District Health Authority Bristol BS2 8EE

Southmead Health Services Trust

E F D MACKENZIE

Southmead Health S

Westbury on Trym

Bristol BS10 5NB

1 Macgregor JE, Campbell MK, Mann EMF, Swanson KY Screening for cervical intraepithelial neoplasia in north east Scotland shows fall in incidence and mortality from invasive cancer with concomitant rise in pre-invasive disease. $B M F$
1994;308:1407-11. (28 May.)

2 Sasieni P. Trends in cervical cancer mortality. Lancet 1991;338: 818-9.

Flannelly G, Anderson D, Kitchener HC, Mann EMF, Campbel $\mathrm{M}$, Fisher $\mathrm{P}$, et al. Management of women with mild and moderate cervical dyskaryosis. BMf 1994;308:1399-403. moderate

4 Hammon $R$. Management of women with smears showing mild dyskaryosis. BMF 1994;308:1383-4. (28 May.)

5 Raffle AE, Alden B, Mackenzie EFD. Six years' audit of laboratory workload and rates of referral for colposcopy in a cervical screening programme in three districts. $B M \mathcal{F} 1990$; 301:901-11.

\section{Long acting methods of contraception}

EDITOR,-North Derbyshire family planning clinics have implanted the six capsule subdermal contraceptive implant, which releases levonorgestrel, into 35 women. The clinics have trained 10 general practitioners and nine family planning doctors in this procedure, and each of these doctors has inserted two or three implants under supervision. Demand will probably be much greater than our ability to purchase the drug. A $£ 15000$ non-recurrent investment in the drug by the health authority will meet the needs of only 72 women as family planning clinics pay value added tax on the basic NHS price of $£ 179$; value added tax is not paid on general practitioners' prescriptions. Is this equitable?

If the current demand continues our stocks will be used up half way through the current financial year. What suggestions do colleagues have for gaining recurrent funding for this sustained release contraceptive other than collating details of the unmet demand and of the numbers on waiting lists for the implant or quoting from The Real Cost of Norplant ${ }^{1}$ and other articles, such as the editorial by Lawrence Mascarenhas'?

The other issue for providers and purchasers constrained by costs is whether the manufacturers could reduce the drug costs by allowing providers to supply their own insertion kits and subtract the cost of these from the price of the drug. This would allow us to purchase more of the drug for more women from the same budget.

E S SEARLE Senior medical officer (family planning)

Saltergate Health Centre,

Saltergate,

Chesterfield S40 1SX

1 Roussel Laboratories? The real cost of Norplant-An analysis of the relative costs of Norplant and alternative forms of hormonal relative costs of Norplant and alternative forms of $h$ contraception. Uxbridge: Roussel Laboratories, 1993.
cos

2 Mascarenhas L. Long acting methods of contraception. $B M F$ 1994;308:991-2. (16 April.)

\section{Upper abdominal pain in pregnancy}

\section{Immediate admission is inappropriate}

EDIToR,-Christopher Barry and colleagues recommend that women who present with epigastric pain and tenderness in pregnancy should be admitted to hospital immediately for exclusion of pre-eclampsia even if they do not have hypertension or proteinuria.' In two of the cases that they report the general practitioner initially diagnosed the presenting symptom of epigastric pain as indigestion.

In my general practice 169 women delivered in 1993. Sixty eight of them had indigestion sufficient to require antacids, and three also required advice for "rib splay." The number with rib splay is an underestimate as the midwives consider this condition to be so common that they often fail to record it. I question the appropriateness of a recommendation that could result in up to $42 \%$ of women being admitted to hospital for exclusion of normotensive, aproteinuric pre-eclampsia at some stage in their pregnancy.

SARAH WOOKEY

West Bar Surgery, SARAH WOOKEY

Banbury,

Oxfordshire OX16 9SF

1 Barry C, Fox R, Stirrat G. Upper abdominal pain in pregnancy may indicate pre-eclampsia. $B M 7$ 1994;308:1562-3. (11 June.)

\section{Measure blood pressure and analyse urine before admission}

EDITOR,-Christopher Barry and colleagues suggest that measurements of blood pressure cannot be relied on to exclude pre-eclampsia and that women with epigastric pain during pregnancy should be admitted to hospital. ${ }^{1}$ Their recommendation was based on the rare occurrence of the syndrome of haemolysis, raised liver enzyme activity, and low platelet count, which may be associated with rapid clinical deterioration. They discuss three cases of upper abdominal pain in pregnancy in women who were later found to have proteinuric hypertension, moderate thrombocytopenia, and abnormal results of liver function tests but who did not have evidence of haemolysis. Therefore, strictly speaking, none of the women had the syndrome of haemolysis, raised liver enzyme activity, and low platelet count.

The authors report two cases of epigastric pain and one of pain in the right upper quadrant, which 